\title{
Distributed Call Admission Control for DESYNC-TDMA in Mobile Ad Hoc Networks
}

\author{
Bongsoo Roh, Myoung-hun Han, Mijeong Hoh, Hwi-Sung Park Kwangsoo Kim, Byeong-hee Roh \\ The $2^{\text {nd }} R \& D$ institute \\ ADD, Daejeon, Republic of Korea \\ \{saintroh, mengddor, hmj, 7hwisung7\} @add.re.kr \\ Department of Computer Engineering \\ Ajou University, Suwon, Republic of Korea \\ \{zubilan, bhroh\}@ajou.ac.kr
}

\begin{abstract}
DESYNC-TDMA is a self-organizing algorithm based on bioinspired features used to implement a collision-free MAC. It does not require a global clock or other infrastructures, and it automatically adjusts to the number of participating nodes to ensure that the available bandwidth is always fully utilized. In DESYNCTDMA the time slots assigned to each node are equally allocated according to the number of nodes, and its slots are allocated one over the number of nodes. It is hard to guarantee the certain level of QoS for certain types of traffic. In this paper, we propose Distributed Call Admission Control (D-CAC) for DESYNC protocol, which is able for nodes to control joining the network with distributed manner. Our scheme needs to add only small amount of bit/flag information to the fire messages of DESYNC protocol. Simulation results showed that D-CAC is able to guarantee a certain amount of allocated slots to send its traffic by control not to join the network if congested.
\end{abstract}

\section{Categories and Subject Descriptors}

C.2.1 [Computer Communication Networks]: Network Architecture and Design - distributed networks, Wireless Communication

\section{General Terms}

Algorithms, Design, Performance

\section{Keywords}

Desynchronization, MANET, TDMA, Call Admission Control

\section{INTRODUCTION}

Mobile Ad Hoc Networks (MANETs) consists of mobile wireless nodes which communicates without centralized administration. Unlike the way of the centralized management of wireless resources, the nodes in MANETs have capabilities of selforganizing, and every node in the network should manage their resource allocation with distributed and cooperative manner. The efficiency of the resource allocation affects the performance of network at MAC layer in distributed environments. Due to the characteristics of MANETs, CSMA/CA mechanism does not guarantee the stable allocation of resources for VoIP traffic which has real time characteristics in multi-hop relay network. In contrast, TDMA mechanism provides a collision-free slot allocation theoretically, and its scheduling scheme guarantees stable performance in the QoS aspect. In general, the complexity and cost of maintaining schedules in TDMA can often outweigh the benefits of fairness, reliability, and high throughput, in the point of view of node and traffic changes. Hence, the sensor nodes usually use the CSMA protocol as the default MAC protocols [1][2].

DESYNC-TDMA [3] is proposed initially for sensor network. Each node distributes its fire message into the cycle and thus all nodes can have same amount of slots by allocating from/to the middle of fires. It can achieve high throughput while guaranteeing fairness among nodes. However, ensuring equal length of slots can lead to allocate insufficient bandwidth to each node when the number of nodes increases in the network. Thus the admission control scheme for DESYNC protocol is required in order to guarantee the slot length of the prior assigned nodes.

In this paper we propose the adaptive Distributed Call Admission Control (D-CAC) scheme for desynchronization protocol which its joining the network is controlled by each joining node. With our DCAC scheme existing nodes in the network can keep their throughput effectively. There is no need of global clock or infrastructure to maintain desynchronization, like original DESYNC algorithm, and it requires minimal overhead for managing bit/flag information. In this paper we focus on guaranteeing the QoS level (i.e. throughput) in the MAC layers.

The paper is organized as follows. Section 2 presents a brief description of DESYCN-TDMA, and Section 3 explains the DCAC algorithm, our proposal. We show the throughput is guaranteed by D-CAC algorithm in Section 4, and we conclude this paper in Section 5.

\section{DESYNC-TDMA Overview}

DESYNC-TDMA [3] is a biologically-inspired primitive based on mutual synchronization of firefly flashing, it is proposed as a MAC protocol for sensor networks. DESYNC-TDMA uses a PulseCoupled Oscillators (PCO) as a resource allocation scheme. Each node adjust its internal reference with Firing Message (FM) and the oscillator firing times are evenly distributed. Every node fires at its firing time which is calculated in a distributed manner as a midpoint between firing times of adjacent neighbor nodes in the previous cycle. TDMA is a common multiplexing scheme for communication that provides each node with a fixed duration transmission slot. So TDMA is rather convenient and implies a global synchronization framework. There are 'global synchronization' and 'empty slot' problem in TDMA-based MANET. However DESYNC-TDMA provides collision-free resource scheduling among nodes with simple desynchronization mechanism in distributed networks. Because DESYNC-TDMA is proposed as a lightweight and effective scheme for providing collision-free TDMA scheduling which aimed at fair allocation of resources for each nodes and reduce the complexity of TDMA by simple self-organizing algorithm. Call Admission Control (CAC) is the process of regulating mechanism particularly in wireless 
mobile networks. CAC can be used to ensure a certain level of QoS or a certain level of performance. DESYNC-TDMA does not provide $\mathrm{CAC}$ for resource allocation, resources are evenly allocated among nodes which shares wireless network as (1) in DESYNCTDMA based MANETs.

As time slots which are allocated to the nodes are affected by the number of nodes in the network, when the nodes are participating in the network increases the amount of resource allocation for individual node decreases. So the network will be crowded. The adaptive algorithm is required to address this problem. When the node is trying to join the network, proposed D-CAC algorithm does not accept the node unconditionally. It control overall network performance by regulating the total number of nodes in the network based on the status of local network with distributed CAC scheme. If a defined limit of QoS is reached, the network cannot accept a new joining node.

The original DESYNC [3] did not support the multihop environment. To support the multihop environment, every node should share its neighbor information within 2 hops and it has to move its fire considering the node 2 hop away. The detailed firing process exchanging the neighbor information was proposed in [4]. Note that our scheme supports multihop extension of DESYNC.

\section{Distributed CAC for DESYNC-TDMA}

In this section, we propose Distributed Call Admission Control scheme for DESYNC-TDMA, which controls for a new node to access the network. We use $Q$ factor which reflects congestion status of a node in decentralized local networks.

We define the $Q$ factor which is a quantifier of MAC congestion. Let $u=\{1,2,3, \ldots, n\}$ denote the index of nodes and $t=\{0,1,2, \ldots$, $k$ \} denote the index of the MAC frames. The $Q$ factor of node $u$ at frame $t, q(u, t)$, is defined as follows:

$$
q(u, t)=\frac{\text { avg }\left\{\text { QueuePacketByte }_{\text {total }}(u)\right\}}{\text { TimeSlotByte }(u, t)}
$$

where QueuePacketBytetotal $(u)$ is the total size of data packets queued at node $u$ and TimeSlotByte $(u, t)$ is the total size of slots allocated to node $u$ at frame $t$. The $q(u, t)$ which is greater than 1 means the allocated slot for node $u$ at frame $t$ is insufficient for the data to transmit. This quantifier could be used to determine whether node $u$ is in the congestion or not. To prevent from fluctuation we applied weighted moving average of $q(u, t)$ as $Q(u, t)$, is written by:

$$
Q(u, t)=\frac{\sum_{i=0}^{k} w_{i} q(u, t-i)}{\sum_{i=0}^{k} w_{i}}
$$

where $w_{i}$ is the weighted value and $k$ is the range parameter.

If $Q(u, t)$ is larger than the threshold, the node $u$ sets NCS flag as 1 and broadcasts NCS information via Firing Message. The NCS $(u, t)$ is defined as follows:

$$
N C S(\mathrm{u}, \mathrm{t})= \begin{cases}1, & \text { if } Q(u, t)>Q_{\text {threshold }} \\ 0, & \text { otherwise }\end{cases}
$$

Now, we define the $N C S_{\text {ratio }}$ as the ratio of the count of nodes having 1 of the NCS flag among received Firing Messages to the number of neighbor nodes in 2 hop range. The $\operatorname{NCS}_{\text {ratio }}(u, t)$ is defined as follows:

$$
N C S_{\text {ratio }}(u, t)=\frac{\sum N C S(N 2(u), t)}{\# N 2(u)}
$$

where $N 2(u)$ denotes the index of neighbor nodes at node $u$ within 2 hop range, and \#N2 $(u)$ denotes the number of neighbors at node $u$ within 2 hop range.
By using the $N C S_{\text {ratio }}(u, t)$ and the $N C S_{\text {threshold }}$, each node including a new joining node could infer the congestion of the network. With this scheme existing node can confirm the joining request if possible (means not congested), and the joining node can wait for mitigating the congested network if congested.

The procedure of D-CAC algorithm is as follows:

1. A new joining node sets Joining Request (JR) as 1.

2. The join node broadcasts Firing Message (FM) including JR after listening a super-frame.

3. Each node which received FM with JR bit as 1, determines the joining message is confirmed or not, by using $N C S_{\text {ratio }}(u, t)$.

A. If the node is confirmed due to the not congested environment, then the node includes the neighbor information in its FM by set Joining Confirm (JC) bit as confirmed (1).

B. Otherwise, the node exclude the request.

4. The joining node receives FM from other nodes.

A. If all fires having its fire with JC in the neighbor list, the joining node joined the network, successfully.

B. Else if there were one or more missing its node information in the FM, then it regards as rejected.

5. The rejected node can choose two below:

A. Idle and wait until the $N C S_{\text {ratio }}$ lower than the $N C S_{\text {threshold. }}$

B. Find another network which it could join on.

\section{Simulation Results}

To show our scheme guarantee the throughput (stands for QoS in here) we simulate our scheme and compare the existing DESYNC scheme. We implement a simulator using Riverbed Modeler (formerly OPNET Modeler). There are 20 nodes and every node initiates one by one every 10 seconds. Every node generates a traffic with an interval of constant (0.01) second and size of 2240 bit/packet. The $\mathrm{k}$ value is set as 5 , and each $q(u, t)$ is equally weighted as 1 . The $Q_{\text {threshold }}$ and $N C S_{\text {threshold }}$ are set as 1 and 0.5, respectively.

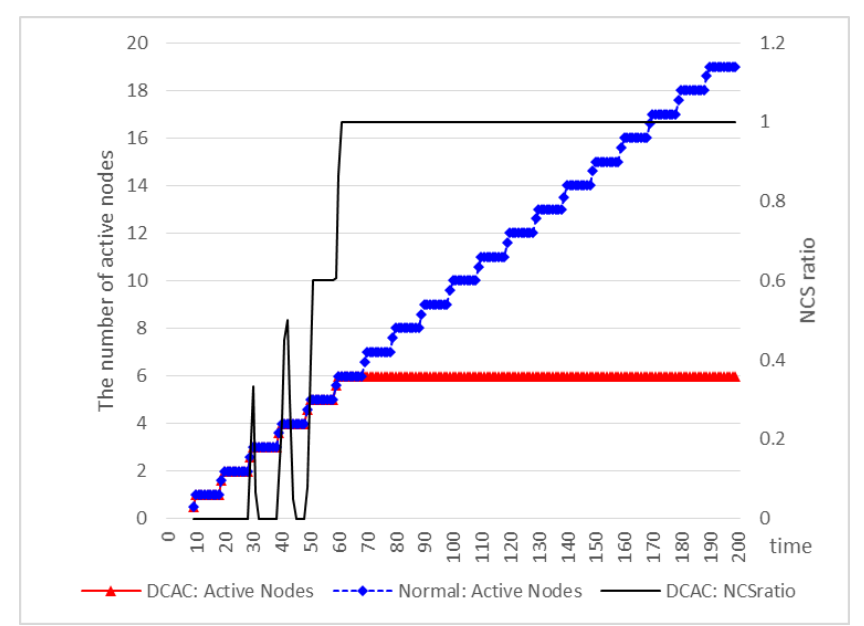

Figure 1. The number of active nodes in DCAC and normal DESYNC according to the NCS ratio.

Figure 1 shows the number of active nodes, which has its slot and actively fires in the network, according to the lapse of time. The graphs for active nodes use left axis, and the $N C S_{\text {ratio }}$ uses right axis. The blue diamond graph of DESYNC without DCAC shows increasing number of nodes in the fire process which makes network more congested. In contrast, the red triangle graph which is our scheme shows the number of active nodes are restricted 
according to the $N C S_{\text {ratio. }}$ If the $N C S_{\text {ratio }}$ is greater than the $N C S_{\text {threshold }}$ (set to 0.5 ,) the joining process is held and the number of active nodes is fixed.

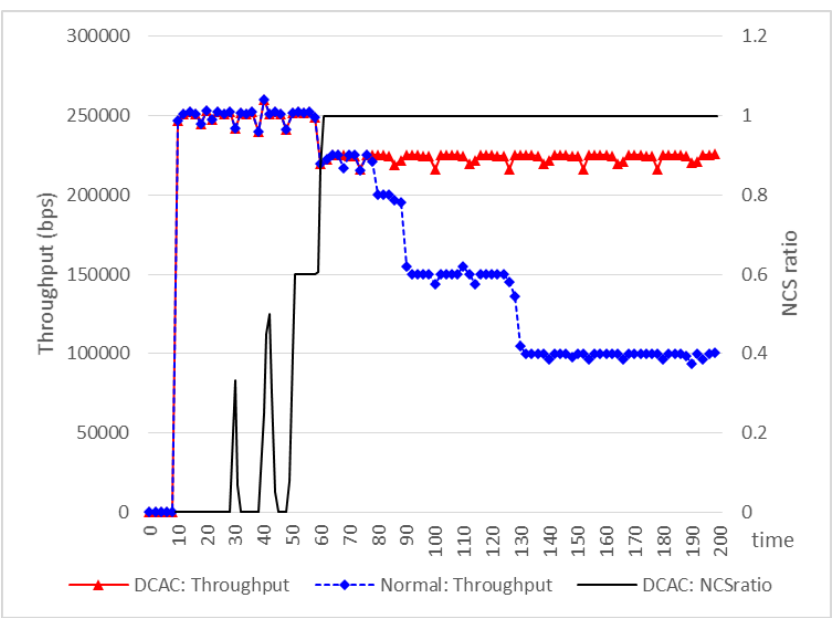

Figure 2. Throughput of DCAC and normal DESYNC according to the NCS ratio.

Related to the Figure 1, Figure 2 shows the comparison of the throughput performance between with and without DCAC scheme. If the NCSratio is greater than the threshold, our scheme can control the joining the node, and can guarantee the level of QoS (in terms of throughput). However, the graph without DCAC show significant degradation of throughput because the more nodes share the bandwidth evenly.

\section{Conclusions}

In this paper, we proposed a D-CAC algorithm for bio-inspired DESYNC-TDMA. It provides a lightweight distributed structure which add small amount of bit/flag information to firing messages of DESYNC. Simulation results showed that D-CAC improved the performance of network compared to the original DESYNCTDMA.

\section{ACKNOWLEDGMENTS}

This work was done at Agency for Defense Development (ADDIBR-245)

\section{REFERENCES}

[1] J. Polastre, J. Hill, and D. Culler, "Versatile low power media access for wireless sensor networks," in SenSys '04, Baltimore, MD, USA, Nov. 2004. pp. 95-107. DOI= http://doi.acm.org/10.1145/1031495.1031508

[2] W. Ye, J. Heidemann, D. Estrin, "An energy-efficient MAC protocol for wireless sensor networks," in INFOCOM '02, New York, NY, USA, Jun. 2002. pp. 1567-1576. DOI= http://dx.doi.org/10.1109/INFCOM.2002.1019408

[3] J. Degesys, I. Rose, A. Patel, and R. Nagpal, "DESYNC: self-organizing desynchronization and TDMA on wireless sensor networks," in IPSN '07, Cambridge, MA, USA, Apr. 2007. pp. 11-20. DOI= http://dx.doi.org/10.1145/1236360.1236363

[4] C. Mühlberger, and R. Kolla, "Extended desynchronization for multi-hop topologies," Institut für Informatik, Universität Würzburg, Tech. Rep 460 (2009). 